> Les cellules souches neurales (CSN) sont des cellules multipotentes, capables de s'autorenouveler et de produire différentes cellules du système nerveux : neurones, astrocytes et oligodendrocytes. La confirmation que la neurogenèse se produit dans des régions discrètes du cerveau des mammifères adultes, l'hippocampe et la région sous-ventriculaire, et que des CSN résident dans le système nerveux central adulte a remis en cause le dogme selon lequel nous naissons avec un nombre limité de neurones, sans possibilité pour le cerveau de se régénérer. Des études récentes ont montré que la neurogenèse est stimulée dans les maladies neurologiques, les accidents vasculaires cérébraux et les traumatismes crâniens, et que des cellules neuronales sont produites aux sites de lésions, où elles remplacent en partie les neurones dégénérés. Dans ces pathologies, la stimulation de la neurogenèse pourrait participer à des phénomènes de neuro-adaptation, et les nouvelles cellules neuronales présentes aux sites de lésions contribueraient à des tentatives de régénération. Cet article résume les travaux étudiant la neurogenèse au cours des pathologies du système nerveux adulte. <

\section{Neurogenèse dans les pathologies du système nerveux}

\author{
Philippe Taupin
}

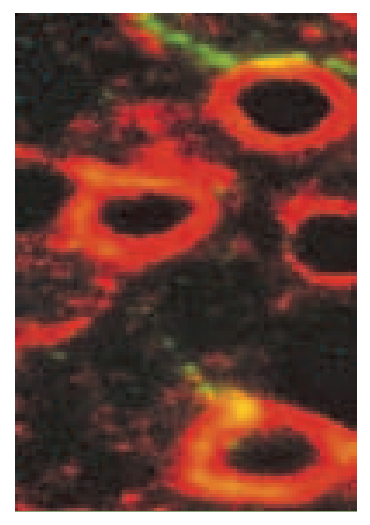

National Neuroscience Institute, 11 Jalan Tan Tock Seng, 308433 Singapour.

National University of Singapore, Nanyang Technological University. philippe_taupin@nni.com.sg

elles se différencient obgpjt@nus.edu.sg en neurones du BO. Ces cellules, qui établissent et reçoivent des connexions fonctionnelles avec les cellules de l'hippocampe et du B0, participeraient aux fonctions physiologiques de ces structures - mémorisation et odorat, mais aussi à la dépression [1, 2].

La neurogenèse se déroulerait également, mais à des taux plus faibles, dans d'autres régions du cerveau adulte comme la corne d'Ammon CAl de l'hippocampe, le cortex et la substance noire (SN), chez différentes espèces $[1,3]$. Cependant, certains de ces résultats ont été contredits et font encore l'objet de débats [ 1 , $4,5]$.

II est probable que la neurogenèse s'effectue chez l'adulte à partir de CSN [6]. Des cellules multipotentes Neurogenèse et cellules souches neurales adultes

Chez les mammifères, et notamment chez l'homme, la neurogenèse se déroule essentiellement dans deux régions du cerveau adulte, le gyrus denté (GD) de l'hippocampe et la partie antérieure de la région sous-ventriculaire (RSV) [1]. Dans le GD, les nouvelles cellules sont produites dans la région sous-granulaire (RSG), puis migrent dans la couche granulaire où elles se différencient en cellules neuronales et gliales. Les nouvelles cellules produites dans la RSV se déplacent, via le courant de migration rostral, vers le bulbe olfactif $(\mathrm{BO})$, où

Article reçu le 22 novembre 2004, accepté le 16 mai 2005. et autorenouvelables ont été isolées et caractérisées, in vitro, à partir de différentes régions du cerveau adulte, dont l'hippocampe et la RSV [1]. Ainsi, des CSN putatives résident dans le cerveau adulte, mais leur source et leur identité doivent encore être déterminées. Les CSN pourraient avoir une origine gliale, et même provenir de cellules progénitrices circulant dans le sang $[1,7]$; l'existence et la potentialité de cette dernière population de cellules progénitices restent toutefois sujettes à discussion [7]. Les CSN expriment des marqueurs tels que la nestine et Musashi 1 $[1,8]$ qui sont, cependant, également exprimées par d'autres types cellulaires, en particulier par des cellules gliales $[1,8]$. 
L'existence d'une neurogenèse et la présence de CSN dans le cerveau adulte ont conduit à étudier leur implication dans le contexte de pathologies du système nerveux. Dans les études rapportées ci-dessous, la neurogenèse a été principalement caractérisée dans les modèles expérimentaux par marquage à la bromodésoxyuridine (BrdU), un marqueur de prolifération cellulaire: la BrdU, analogue de la thymidine, s'insère dans l'ADN des cellules au cours de la phase $S$ du cycle cellulaire $[1,2]$.

\section{Neurogenèse dans les maladies neurologiques}

Des autopsies de patients atteints de la maladie d'Alzheimer (MA) montrent une augmentation, dans la RSG et la couche granulaire du $G D$, de l'expression de marqueurs de cellules neuronales immatures comme la doublecortine et la molécule polysialylée d'adhésion cellulaire des neurones, suggérant que la neurogenèse est augmentée dans I'hippocampe au cours de cette maladie [9]. La caractérisation de la neurogenèse dans des modèles animaux de $M A$, souris déficientes pour les gènes de la préséniline ou du précurseur de la protéine amyloïde, montrent que la neurogenèse y est diminuée dans la RSG et la RSV [10, 11]. Ces animaux présentent une RSV peu développée, ce qui suggère une diminution du nombre de cellules progénitrices lors du développement, entraînant une réduction du nombre de ces cellules chez l'adulte. Ainsi, la divergence des résultats observée entre les autopsies et les modèles animaux pourrait provenir de la limitation de ces modèles pour étudier les maladies complexes, mais aussi les phénotypes adultes. La neurogenèse a récemment été caractérisée chez des souris transgéniques exprimant des mutations de la protéine amyloïde [12]: elle est augmentée dans le GD et la RSV, en accord avec les observations réalisées chez l'homme.

Des autopsies de patients atteints de la maladie de Huntington (MH) montrent que l'expression des marqueurs du cycle cellulaire, dont l'antigène nucléaire de prolifération cellulaire, et des cellules neuronales immatures, dont la $\beta$-tubuline III, est augmentée dans la RSV, et que des cellules neuronales nouvellement produites migrent vers la région endommagée du striatum [13]: la neurogenèse serait donc augmentée dans la RSV au cours de cette maladie. Des études réalisées chez des souris transgéniques mutantes pour le gène de l'huntingtine (souris R6/1, modèles de la $\mathrm{MH}$ ) ont rapporté une diminution de la neurogenèse dans le GD [14]. Dans un modèle de lésion de la $M H$, une augmentation de la neurogenèse dans la RSV, une migration de neuroblastes dans la région endommagée du striatum et la formation de nouveaux neurones ont été également remarquées, appuyant là aussi les résultats observés chez l'homme [15].

D'autres résultats suggèrent que de nouvelles cellules dopaminergiques sont produites dans la SN, chez le rat adulte [3]. Dans cette dernière étude, les auteurs montrent que leur production est multipliée par 2, trois semaines après une lésion induite par la MPTP (1-méthyl-4-phényl-1,2,3,6 tétrahydropyridine). D'après ces données, la production de nouvelles cellules dopaminergiques serait stimulée dans la maladie de Parkinson (MP); la neurogenèse au sein de la SN pourrait alors être une cause de MP, dans le cas où elle ne produirait pas suffisamment de nouveaux neurones pour compenser ceux qui dégénèrent. Cependant, ces conclusions doivent être prises avec précaution, dans la mesure où ces résultats n'ont encore pu être reproduits $[4,5]$.

Dans des modèles animaux d'épilepsie chez le rongeur (crises induites par la pilocarpine, par exemple), la neurogenèse est stimulée bilatéralement dans le GD et la RSV, des cellules ectopiques de type granulaire sont produites dans le hile, jusque dans la région CA3, et des cellules naissantes contribuent au bourgeonnement des fibres moussues dans la couche moléculaire du GD [16, 17]. Ces résultats ont été confirmés dans d'autres modèles d'épilepsie. L'inhibition de la neurogenèse dans le GD, à l'aide d'une faible dose de rayon $X$ sur le cerveau adulte, n'a pas d'effet sur les crises. Ainsi, l'augmentation de la neurogenèse, la production de cellules ectopiques de type granulaire et la synaptogenèse anormale des fibres moussues sont des traits de l'épilepsie, et représentent vraisemblablement des événements indépendants. II reste à déterminer le rôle de chacun dans l'épilepsie, mais aussi de valider ces données chez l'homme.

\section{Neurogenèse dans les accidents vasculaires cérébraux}

Après ischémie cérébrale globale chez le rongeur, le nombre de cellules produites dans la RSG est augmentée par un facteur 12 dans les jours qui suivent l'accident vasculaire cérébral (AVC) [18]. Après 1 mois, ces cellules ont migré vers la couche granulaire, où elles se différencient en cellules neuronales matures exprimant des marqueurs tels que l'antigène nucléaire des cellules neuronales (NeuN), la protéine associée aux microtubules-2 (Map-2), et la protéine se liant au calcium $(\mathrm{CaBP})$. Plusieurs études confirment ces résultats chez les rongeurs et les primates non humains, et montrent que la neurogenèse est stimulée bilatéralement dans la RSG et la RSV après ischémie globale ou focale. Par ailleurs, les cellules produites dans la RSG expriment Musashi 1 [19]. Ainsi, la neurogenèse est stimulée après ischémie cérébrale, et les cellules nouvellement produites ont un phénotype de cellules souches et progénitrices neurales.

Des cellules sont également produites dans les régions de dégénération après ischémie globale et focale, telles que la région CAl de l'hippocampe, le striatum et le cortex, où elles se différencient en cellules neuronales dont le phénotype est semblable à celui des cellules ayant dégénéré [20-22]. Ce remplacement reste marginal, ne représentant qu'un faible pourcentage des neurones dégénérés, de l'ordre de 0,2\% dans le striatum après ischémie (focale) [21]. L'origine de ces cel- 
Iules neuronales dans les régions de dégénération a été déterminée par des études de marquage, en particulier avec des rétrovirus: elles proviennent de la RSV, et empruntent, en partie, le courant de migration rostral pour atteindre leur destination [21, 22].

\section{Neurogenèse dans les traumatismes crâniens}

Dans des modèles de contusions expérimentales chez le rongeur, le niveau d'incorporation de BrdU dans la RSG est, bilatéralement, multiplié par 5,9 dans les jours qui suivent le traumatisme, puis revient à son niveau normal au bout de 15 jours. Après 1 mois, la majorité des cellules marquées par la BrdU ont migré dans la couche granulaire du GD et expriment des marqueurs de cellules neuronales matures comme la CaBP [23]. La neurogenèse est donc stimulée dans le GD après une contusion. Une augmentation du nombre de cellules naissantes a également été rapportée au site de lésion. Un mois après le traumatisme, ces cellules expriment presque exclusivement un phénotype astrocytaire et participeraient principalement à la cicatrice gliale [24]. Une gliogenèse est donc observée après traumatisme crânien. Des marquages rétroviraux montrent que des cellules produites dans la RSV migrent vers le site de lésion, où elles se différencient en cellules gliales [25]; ces résultats n'éliminent cependant pas une origine locale ou périphérique des cellules gliales constituant la cicatrice. Un seul rapport met en évidence la production de nouvelles cellules neuronales au site de lésion [26]: si l'origine de ces cellules n'a pas encore été déterminée, il est probable qu'elles proviennent de la migration de cellules progénitrices neuronales à partir de la RSV, comme cela a été observé dans d'autres modèles de lésions du système nerveux central [13, 15, 20-22]. Il est également possible qu'elles aient une origine locale, voire périphérique. Cependant, dans ce dernier cas, certaines données sont encore sujettes à controverse [7].

\section{Mécanismes de stimulation de la neurogenèse}

Ces mécanismes de stimulation de la neurogenèse dans les pathologies du système nerveux sont peu connus. II est postulé que la mort cellulaire stimule la prolifération des cellules progénitrices dans le cerveau adulte [27]. Dans les maladies neurologiques, les AVC et les traumatismes crâniens, une augmentation de la neurogenèse coïncide avec la mort cellulaire. Cependant, dans des modèles d'épilepsie, la stimulation de la neurogenèse se produit même en présence d'inhibiteurs d'apoptose, comme les caspases, et l'augmentation de la neurogenèse a été inversement corrélée avec la dégénérescence cellulaire [28]. La mort cellulaire ne serait donc pas nécessaire pour stimuler la neurogenèse dans les pathologies du système nerveux, cette stimulation étant probablement régulée par le nombre de cellules progénitrices. La mort cellulaire pourrait ainsi agir séparément, ou en coopération avec d'autres mécanismes, pour solliciter la neurogenèse dans ces pathologies.

L'augmentation bilatérale de la neurogenèse rapportée dans l'épilepsie, les AVC et les traumatismes crâniens pourrait faire intervenir des facteurs trophiques, des régions neurogéniques et du liquide céphalorachidien. La migration des cellules naissantes vers les sites de lésions, dans la maladie de Huntington, les AVC et les traumatismes crâniens, suggère la présence de facteurs, au niveau des lésions, susceptibles d'attirer ces cellules. L'existence de tels facteurs a été mise en évidence dans des études où les cellules souches et progénitrices, greffées ou administrées intravasculairement chez des animaux atteints de tumeurs, lésions cérébrales ou maladies neurologiques, colonisent les régions endommagées du cerveau $[29,30]$. Des mécanismes similaires pourraient intervenir au cours de la migration des cellules naissantes vers les lésions occasionnées par différentes maladies du système nerveux.

\section{Conclusions et perspectives}

Parce que les cellules souches neurales peuvent produire l'ensemble des phénotypes du système nerveux central, leur intérêt majeur repose sur leur utilisation à des fins thérapeutiques. Les résultats présentés ici montrent que la neurogenèse est augmentée dans différentes pathologies du système nerveux, et que de nouvelles cellules neuronales sont produites aux sites de lésions, où elles remplacent une partie des cellules nerveuses dégénérées. Cette augmentation de la neurogenèse, sa contribution et son rôle doivent cependant encore être définis ; la neurogenèse pourrait participer à des phénomènes de neuroadaptation, dans les troubles de la mémoire ou la dépression associés à ces pathologies. La production de cellules neuronales au niveau des lésions pourrait, quant à elle, signifier une tentative de régénération. Leur origine, la région sous-ventriculaire, suggère que des protocoles de stimulation de la neurogenèse dans cette zone pourrait être bénéfique pour la réparation du système nerveux après lésions. $\diamond$

\section{SUMMARY}

Neurogenesis in the pathologies of the nervous system

Neural stem cells (NSCs) are the self-renewing, multipotent cells that generate neurons, astrocytes, and oligodendrocytes in the nervous system. Contrary to the long-held dogma, neurogenesis occurs in discrete areas of the adult brain, the hippocampus and the subventricular zone, and NSCs reside in the adult central nervous system. Recent studies have shown that neurogenesis is increased in the diseased brains, after strokes and traumatic brain injuries, and that new neuronal cells are generated at the sites of injury, where they replace some of the degenerated nerve cells. Thus, the central nervous system has the capacity to regenerate after injury. The contribution and function of the increased neurogenesis in the pathologies of the nervous system 
remain to be understood. The increased hippocampal neurogenesis may play a role in neuroadaptation, such as in memory troubles and depression, associated with these pathologies. The increased neurogenesis at the sites of injury may represent an attempt by the central nervous system to regenerate itself after injury. Newly generated neuronal cells at the sites of injury originate from the subventricular zone. Hence, strategies that would promote neurogenesis in the subventricular zone may promote neuronal repair after injury of the nervous system. In this manuscript, we will review the studies on neurogenesis in the pathologies of the nervous system. $\diamond$

\section{REMERCIEMENTS}

Je remercie D.J. Taupin pour la relecture de ce manuscrit. P. Taupin reçoit le soutien de subventions du NMRC, BMRC et de la Juvenile diabetes research foundation.

\section{RÉFÉRENCES}

1. Taupin P, Gage FH. Adult neurogenesis and neural stem cells of the central nervous system in mammals. J Neurosci Res 2002 ; 69 : 745-9.

2. Taupin P. Contrôle de la persistance des cellules souches neurales des mammifères. Med Sci (Paris) $2004 ; 20: 748-9$.

3. Zhao M, Momma S, Delfani K, et al. Evidence for neurogenesis in the adult mammalian substantia nigra. Proc Natl Acad Sci USA 2003; 100 : 7925-30.

4. Lie DC, Dziewczapolski G, Willhoite AR, et al. The adult substantia nigra contains progenitor cells with neurogenic potential. J Neurosci $2002 ; 22: 6639-49$.

5. Frielingsdorf H, Schwarz K, Brundin P, Mohapel P. No evidence for new dopaminergic neurons in the adult mammalian substantia nigra. Proc Natl Acad Sci USA 2004 ; 101 : 10177-82.

6. Gage FH. Mammalian neural stem cells. Science $2000 ; 287$ : 1433-8.

7. Mezey $\varepsilon$. Commentary on bone marrow stem cells and openmindedness. Stem Cells Dev $2004 ; 13: 147-52$.

8. Kaneko Y, Sakakibara S, Imai T, et al. Musashi 1 : an evolutionally conserved marker for CNS progenitor cells including neural stem cells. Dev Neurosci $2000 ; 22$ : 139-53.

9. Jin K, Peel AL, Mao XO, et al. Increased hippocampal neurogenesis in Alzheimer's disease. Proc Natl Acad Sci USA 2004 ; 101 : 343-7.

10. Feng $R$, Rampon C, Tang YP, et al. Deficient neurogenesis in forebrain-specific presenilin-1 knockout mice is associated with reduced clearance of hippocampal memory traces. Neuron $2001 ; 32: 911-26$ et $2002 ; 33: 313$ (erratum).

11. Haughey NJ, Nath A, Chan SL, et al. Disruption of neurogenesis by amyloid beta-peptide, and perturbed neural progenitor cell homeostasis, in models of Alzheimer's disease. J Neurochem 2002 ; 6 : 1509-24.
12. Jin K, Galvan V, Xie L, et al. Enhanced neurogenesis in Alzheimer's disease transgenic (PDGF-APPSw, Ind) mice. Proc Natl Acad Sci USA 2004 ; 101 : $13363-7$.

13. Curtis MA, Penney $\varepsilon B$, Pearson AG, et al. Increased cell proliferation and neurogenesis in the adult human Huntington's disease brain. Proc Natl Acad Sci USA $2003 ; 100: 9023-7$.

14. Lazic SE, Grote H, Armstrong RJ, et al. Decreased hippocampal cell proliferation in R6/1 Huntington's mice. Neuroreport 2004 ; $15: 811-3$.

15. Tattersfield AS, Croon RJ, Liu YW, et al. Neurogenesis in the striatum of the quinolinic acid lesion model of Huntington's disease. Neuroscience $2004 ; 127$ : 319-32.

16. Parent JM, Yu TW, Leibowitz RT, et al. Dentate granule cell neurogenesis is increased by seizures and contributes to aberrant network reorganization in the adult rat hippocampus. J Neurosci $1997 ; 17: 3727-38$.

17. Parent JM, Tada $\varepsilon$, Fike JR, Lowenstein DH. Inhibition of dentate granule cell neurogenesis with brain irradiation does not prevent seizure-induced mossy fiber synaptic reorganization in the rat. J Neurosci 1999 ; $19: 4508-19$.

18. Liu J, Solway K, Messing RO, Sharp FR. Increased neurogenesis in the dentate gyrus after transient global ischemia in gerbils. J Neurosci $1998 ; 18$ : 7768-78.

19. Yagita Y, Kitagawa K, Ohtsuki T, Takasawa Ki. Neurogenesis by progenitor cells in the ischemic adult rat hippocampus. Stroke $2001 ; 32: 1890-6$.

20. Nakatomi H, Kuriu T, Okabe $S$. Regeneration of hippocampal pyramidal neurons after ischemic brain injury by recruitment of endogenous neural progenitors. Cell 2002; $110: 429-41$.

21. Arvidsson A, Collin T, Kirik D, et al. Neuronal replacement from endogenous precursors in the adult brain after stroke. Nat Med $2002 ; 8: 963-70$.

22. Jin K, Sun Y, Xie L, et al. Directed migration of neuronal precursors into the ischemic cerebral cortex and striatum. Mol Cell Neurosci 2003 ; 24 : 171-89.

23. Dash PK, Mach SA, Moore AN. Enhanced neurogenesis in the rodent hippocampus following traumatic brain injury. J Neurosci Res $2001 ; 63$ : 313-9.

24. Kernie SG, Erwin TM, Parada LF. Brain remodeling due to neuronal and astrocytic proliferation after controlled cortical injury in mice. J Neurosci Res 2001; 66: 317-26.

25. Goings GE, Sahni V, Szele FG. Migration patterns of subventricular zone cells in adult mice change after cerebral cortex injury. Brain Res 2004 ; $996: 213-26$.

26. Braun H, Schafer K, Hollt V. Beta III tubulin-expressing neurons reveal enhanced neurogenesis in hippocampal and cortical structures after a contusion trauma in rats. J Neurotrauma $2002 ; 19: 975-83$.

27. Gould $\varepsilon$, Tanapat $P$. Lesion-induced proliferation of neuronal progenitors in the dentate gyrus of the adult rat. Neuroscience $1997 ; 80: 427-36$.

28. Ekdahl CT, Mohapel P, Elmer $\varepsilon$, Lindvall 0 . Caspase inhibitors increase shortterm survival of progenitor-cell progeny in the adult rat dentate gyrus following status epilepticus. EurJ Neurosci $2001 ; 14$ : 937-45.

29. Aboody KS, Brown A, Rainov NG, et al. Neural stem cells display extensive tropism for pathology in adult brain : evidence from intracranial gliomas. Proc Natl Acad Sci USA 2000; $97: 12846-51$ et 2001 ; $98: 777$ (erratum).

30. Macklis JD. Transplanted neocortical neurons migrate selectively into regions of neuronal degeneration produced by chromophore-targeted laser photolysis. J Neurosci $1993 ; 13: 3848-63$.

\section{TIRÉS À PART}

P. Taupin

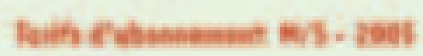

Abonnez-vous

à Médecine/Sciences
$>1985-2005$, depuis 20 ans, grâce à $\mathrm{m} / \mathrm{s}$, vous vivez en direct les progrès des sciences biologiques et médicales

Ihlletin o'cotanerenent

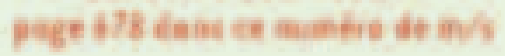
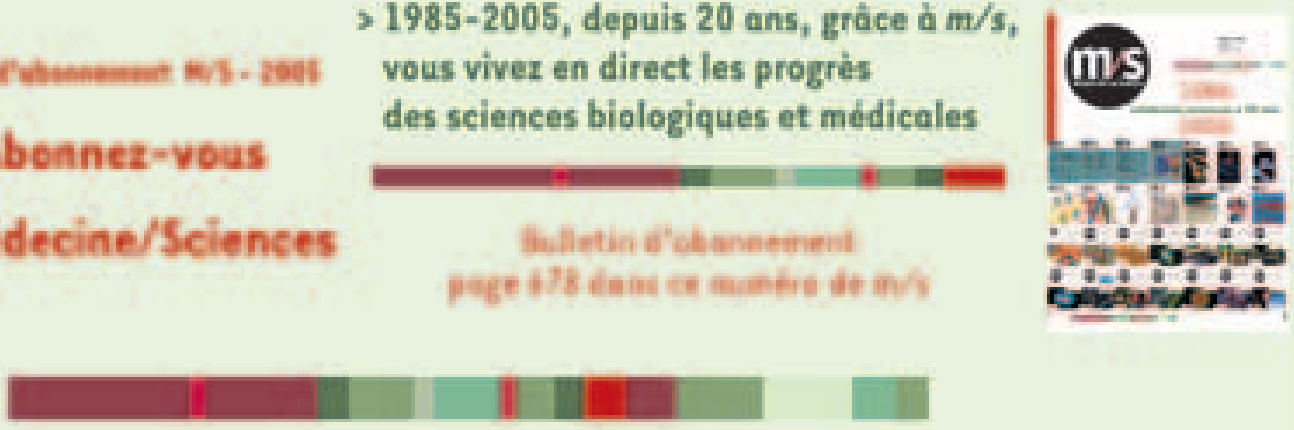